\title{
Leaking rheumatoid olecranon bursitis as a cause of forearm swelling
}

\author{
J. D. MACFARLANE AND SJ VAN DER LINDEN
}

From the Department of Rheumatology, University Hospital, 2333 AA Leiden, The Netherlands

SUMMARY A patient with seropositive rheumatoid arthritis and recurrent oedema of the forearm was shown to have a leak from a multilobulated olecranon bursa.

Bursal swelling is well known in rheumatoid arthritis and is particularly common at the elbow, ${ }^{1}$ where it is usually of little clinical consequence unless infection supervenes. To our knowledge rupture of an olecranon bursa has not been previously reported.

\section{Case report}

A 76-year-old man with recurrent asthmaticobronchitis, bronchiectasis, and seropositive, erosive rheumatoid arthritis of 6 years' duration was reasonably well controlled on salbutamol, indomethacin, and gold therapy. In March 1979 he developed pain and swelling in the right forearm, the swelling being greatest every evening.

Clinical examination in April 1979 showed diffuse swelling of the right forearm extending proximally from the wrist for about $10 \mathrm{~cm}$. An olecranon bursa was present bilaterally. A definite cyst was palpable on the volar aspect of the right wrist, which, like the elbow joint, showed synovial swelling and some limitation of movement.

Conventional radiographs showed erosive changes in both elbows and wrists. Echography of the right forearm suggested small fluid-containing areas compatible with a leak from a cyst. Arthrography of the radiocarpal joint failed owing to inability to enter the distorted joint space. Renal and cardiac function was normal.

There was initially some spontaneous improvement in the arm swelling, but he returned in August 1979 with pitting oedema in both forearms. The rest

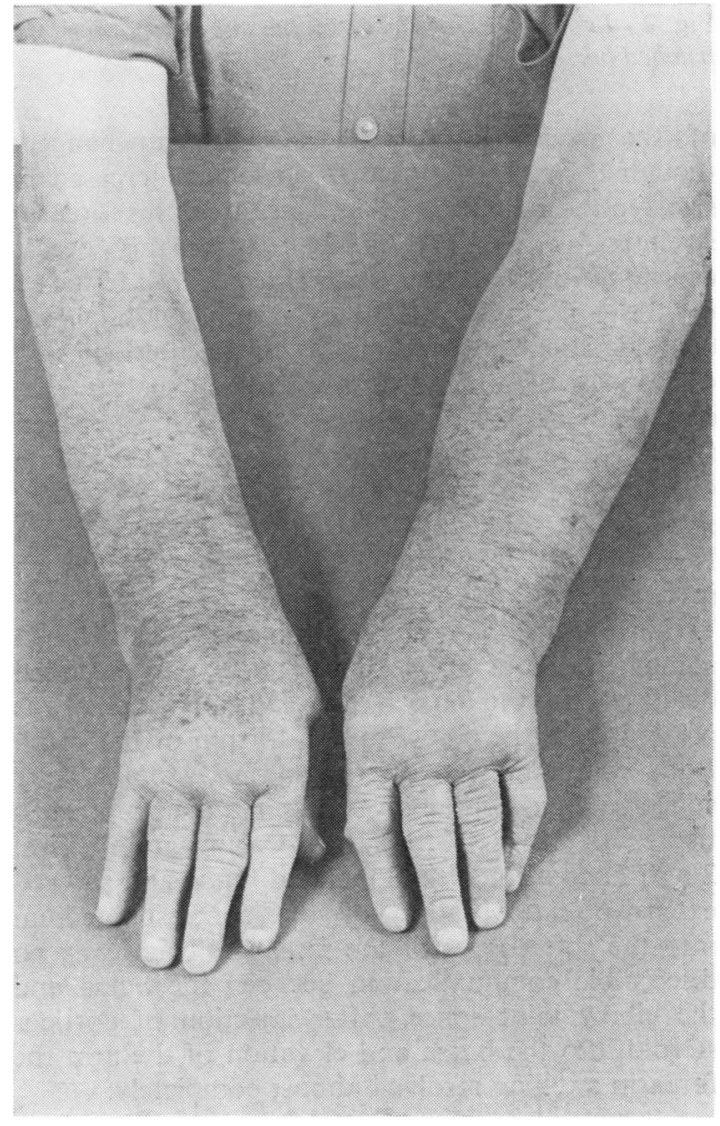

Fig. 1 Clinical photograph showing marked swelling of left forearm. 


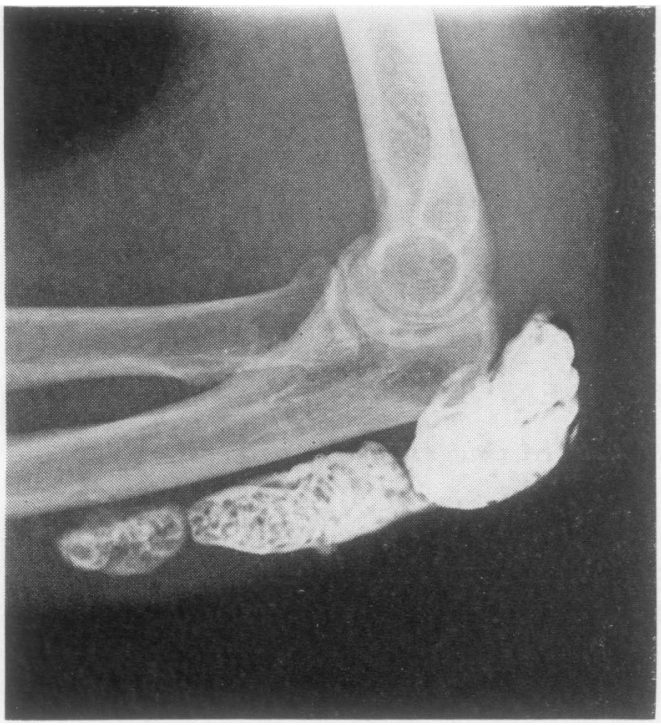

Fig. 2 Lateral view of olecranon bursagram showing trilobed bursa.

of the examination was essentially unchanged, though the right olecranon bursa had increased in size. On the assumption that the cause of his forearm swelling was possibly a leak from this bursa, aspiration of the bursa was performed and followed by the injection of $1 \mathrm{ml}$ of triamcinolone hexacetonide (Lederspan). Analysis of the fluid showed 4100 cells of which $91 \%$ were polynuclear. No crystals or organisms were seen and cultures proved sterile. There was a prompt reduction in the swelling of the right forearm. However, the left forearm swelling persisted and worsened. When seen in November 1979 the clinical appearance was as shown in Fig. 1.

Fluctuation was demonstrable between all 3 compartments of an olecranon bursa that extended distally along the ulnar border. ${ }^{2}$ Pitting oedema was present from the elbow to the dorsum of the hand. Both echography and computerised tomography suggested fluid-containing spaces in the subcutaneous tissues of the left forearm. A bursagram (Figs. 2 and 3) confirmed the lobulated bursa and showed extensive extravasation of the contrast medium into the soft tissues of the forearm. There was no discernible communication between the bursa and the elbow joint space. After injection of corticosteroid into the bursa and elevation of the arm the forearm swelling resolved almost completely.

\section{Discussion}

The clinical feature of recurrent oedematous swelling

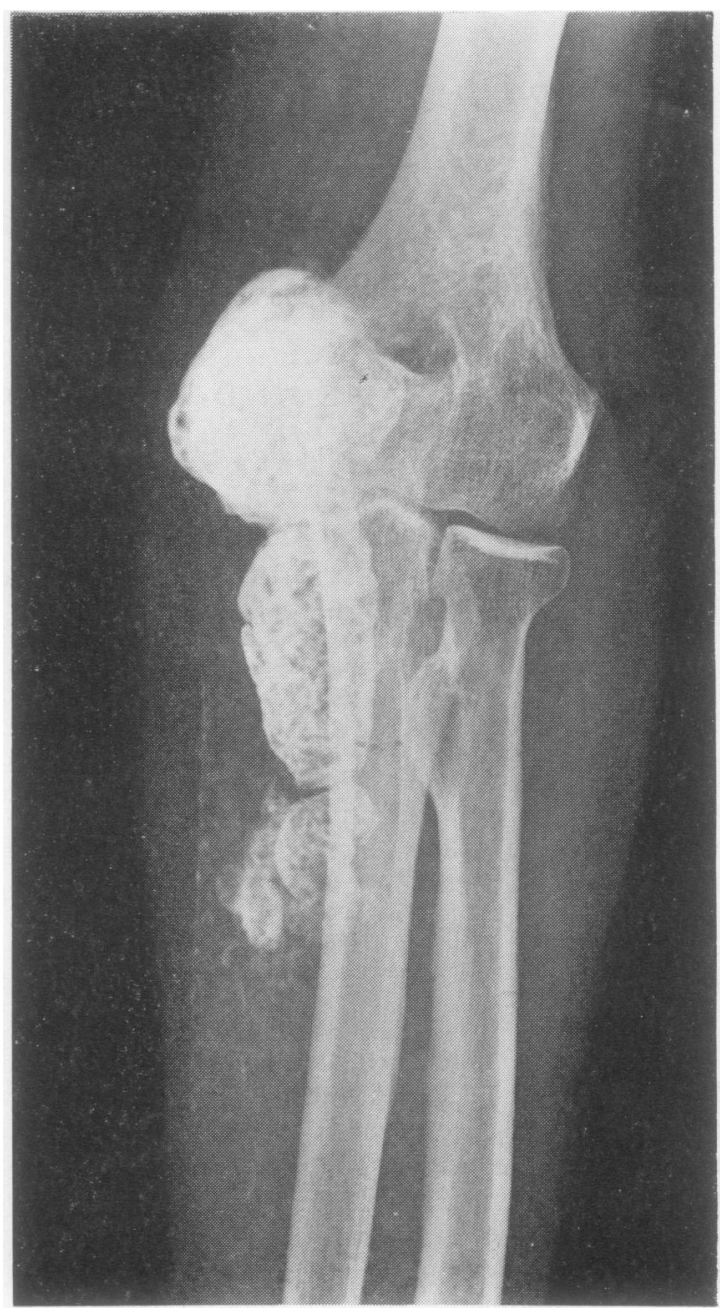

Fig. 3 Anteroposterior view of olecranon bursagram showing widespread leakage of contrast material in the soft tissues.

confined to the forearms suggested a local cause, and, though synovial rupture seemed likely, especially in the first episode when an cystic swelling was identified near the wrist, ${ }^{34}$ this seemed an inadequate explanation for the later events. There was no clinical or radiological evidence for any other cause of local oedema in the arm, such as fracture, lymphoedema, or obstruction of superior vena cava.

The demonstration of bilateral olecranon bursae with reduction of forearm swelling after bursal corticosteroid injection and the radiographic finding of contrast in the soft tissues after bursography lend 
credence to the idea that leakage from an inflammatory bursitis was responsible for the clinical picture. This situation is somewhat analogous to the chronic leak from a popliteal cyst as described by Myles, ${ }^{5}$ but in our patient no connection between the bursa and the joint was found.

The help of the Departments of Radiology and Medical Illustration is greatly appreciated.

\section{References}

1 Hollander J L, McCarty D J, eds. Arthritis and Allied Conditions. Philadelphia: Lea and Febiger, 1972: 1403-4.

2 Gerber N J, Dixon A St J. Synovial cysts and juxtaarticular bone cysts (geodes). Semin Arthritis Rheum 1974; 3: 323-48.

3 Jayson M I V, Swannell A J, Kirk J A, Dixon A St J. Acute joint rupture. Ann Phys Med 1969; 10: 175-9.

4 Iveson J M I, Hill A G S, Wright V. Wrist cysts and fistulae. Ann Rheum Dis 1975; 34: 388-94.

5 Myles A B. Posterior synovial leaks in arthritis of the knee. Proc R Soc Med 1971; 64: 262-4. 